TRANSACTIONS OF THE

AMERICAN MATHEMATICAL SOCIETY

Volume 355, Number 5 , Pages 1905-1920

S 0002-9947(03)03090-3

Article electronically published on January 10, 2003

\title{
MAPPINGS OF FINITE DISTORTION: THE SHARP MODULUS OF CONTINUITY
}

\author{
PEKKA KOSKELA AND JANI ONNINEN
}

\begin{abstract}
We establish an essentially sharp modulus of continuity for mappings of subexponentially integrable distortion.
\end{abstract}

\section{INTRODUCTION}

We consider mappings $f \in W_{\text {loc }}^{1,1}\left(\Omega, \mathbb{R}^{n}\right)$, where $\Omega \subset \mathbb{R}^{n}$ is a domain. Such a mapping is said to have finite distortion if the following three conditions are satisfied:

(1) $f \in W_{l o c}^{1,1}\left(\Omega, \mathbb{R}^{n}\right)$.

(2) The Jacobian determinant $J(x, f)$ of $f$ is locally integrable.

(3) There is a measurable function $K_{O}=K_{O}(x) \geq 1$, finite almost everywhere, such that $f$ satisfies the distortion inequality

$$
|D f(x)|^{n} \leq K_{O}(x) J(x, f) \quad \text { a.e. }
$$

Notice that when $K_{O} \in L^{\infty}$, we recover the class of mappings of bounded distortion, also called quasiregular mappings. In this case, $f$ is locally Hölder continuous with exponent $K_{O}^{-1}$, and this exponent is sharp. By this we mean that $f$ has such a representative; this comment also applies to what follows. Recently, (non-constant) mappings of finite distortion with subexponentially integrable distortion $K_{O}$ have been shown to share many of the nice properties of mappings of bounded distortion, such as being open and discrete [5], [12], [14]. Here the subexponential integrability of $K_{O}$ requires that

$$
\exp \left(\mathcal{A}\left(K_{O}\right)\right) \in L_{\text {loc }}^{1}(\Omega)
$$

with $\mathcal{A}$ smooth and strictly increasing, with

(i) $\int_{1}^{\infty} \frac{\mathcal{A}^{\prime}(t)}{t} d t=\infty$, and so that

(ii) there exists $t_{0} \in(0, \infty)$ such that $\mathcal{A}^{\prime}(t) t$ increases to infinity for $t \geq t_{0}$.

In particular, under this assumption, a mapping $f$ of finite distortion is continuous. The integrability assumption on $K_{O}$ is sharp as regards continuity (and openness and discreteness) in the sense that mappings of finite distortion with

Received by the editors January 25, 2002.

2000 Mathematics Subject Classification. Primary 30C65.

The authors were supported in part by the Academy of Finland, project 39788. The second author was also supported by the foundations Magnus Ehrnroothin Säätiö and Vilho, Yrjö ja Kalle Väisälän Rahasto. This research was completed when the authors were visiting at the University of Michigan, Pekka Koskela as the Fred and Lois Gehring professor. They wish to thank the Institute for the hospitality. 
$\exp \left(\mathcal{B}\left(K_{O}\right)\right) \in L_{\text {loc }}^{1}(\Omega)$ and without a continuous representative can be constructed whenever $\int_{1}^{\infty} \frac{\mathcal{B}^{\prime}(t)}{t} d t<\infty$, see [14].

Let us consider the case $\exp \left(\lambda K_{O}\right) \in L_{l o c}^{1}(\Omega)$. By the results in [7], $f$ then has (locally) a modulus of continuity of the type

$$
|f(x)-f(y)| \leq C / \log \log (R /|x-y|) .
$$

It is also known that better bounds can be obtained when the constant $\lambda$ is sufficiently large: given $s>0$, the (local) modulus of continuity

$$
|f(x)-f(y)| \leq C(\log (R /|x-y|))^{-s}
$$

holds provided $\lambda \geq \lambda(s, n)$. This conclusion is established in [6] as a corollary to a surprising regularity property of mappings with exponentially integrable distortion that does not hold for small $\lambda$. In this paper we establish an essentially sharp modulus of continuity by a different technique.

Theorem 1.1. Let $f: \Omega \rightarrow \mathbb{R}^{n}$ be a mapping of finite distortion whose distortion function $K_{O}$ satisfies the integrability condition

$$
K:=\int_{B} \exp \left(\lambda K_{O}(x)\right) d x<\infty,
$$

where $\lambda>0$ and $B=B\left(x_{0}, R\right) \subset \subset \Omega$. Then for every small $\epsilon>0$ and all $x, y \in B\left(x_{0},\left(\frac{R}{8}\right)^{e}\left[\frac{n}{\omega_{n-1}} K\right]^{\frac{1-e}{n}}\right)$, we have the estimate

$$
|f(x)-f(y)| \leq C_{K, R, n, \lambda}(\epsilon) \frac{\left(\int_{B} J(z, f) d z\right)^{\frac{1}{n}}}{\log ^{\frac{\lambda}{n}-\epsilon}\left(\frac{n K}{\omega_{n-1}|x-y|^{n}}\right)} .
$$

Here $\omega_{n-1}$ is the surface measure of $\partial B(0,1)$.

The following example shows that our modulus of continuity is essentially sharp.

Example 1.2. Let $\lambda>0$. Then there exist a continuous mapping $f: B(0,1) \rightarrow \mathbb{R}^{n}$ of finite distortion such that

$$
\int_{B(0,1)} \exp \left(\lambda K_{O}(x, f)\right) d x<\infty
$$

and points $x_{j}, y_{j} \in B(0,1)$ so that $x_{j} \rightarrow 0, y_{j} \rightarrow 0$ and

$$
\left|f\left(x_{j}\right)-f\left(y_{j}\right)\right| \log ^{\frac{\lambda}{n}+\epsilon}\left(\frac{1}{\left|x_{j}-y_{j}\right|}\right) \rightarrow \infty
$$

for every $\epsilon>0$.

We believe that the claim of Theorem 1.1 holds with the exponent $\lambda / n$, but we have not been able to verify this.

Theorem 1.1 is new even for homeomorphic solutions to the Beltrami equation in the plane. By the results in [1] and 9], one obtains a logarithmic modulus of continuity of the type given in Theorem 1.1 for solutions to the Beltrami equation but with a worse exponent.

By the discussion above and Theorem 1.1 one would expect an essentially sharp modulus of continuity even under the weaker assumption that exp $\mathcal{A}\left(K_{O}\right) \in L_{\text {loc }}^{1}(\Omega)$ with $\mathcal{A}$ satisfying (i) and (ii). This is indeed the case, and such a result will be given in Section 4. 
Let us close this introduction by briefly sketching the proof of Theorem 1.1 We mimic the lines of reasoning by Morrey [16] and Reshetnyak [18] for the case of mappings of bounded distortion as follows. We prove a decay estimate on the integrals of the Jacobian of $f$ over balls by establishing a differential inequality for these integrals. This is done by employing a suitable isoperimetric inequality established in [17] (also see [8]); the crucial point behind the isoperimetric inequality is that, under our assumptions, the pointwise Jacobian of $f$ coincides with the distributional Jacobian. In the (classical) case of mappings of bounded distortion, the decay estimate on the Jacobian guarantees that $f$ belongs to a Morrey class and is thus Hölder continuous [16], [18, [10]. In our setting, the decay order is too weak to imply even continuity for a general mapping. To bypass this difficulty, we insert

the fundamental ideas from our initial proof of continuity in [7] to show that, for our mappings, the decay order suffices to yield the desired modulus of continuity.

\section{ORLICZ SPACES}

Theorem 1.1 will be obtained as a corollary to a more general result. Let us replace the assumption $\exp \left(\lambda K_{O}\right) \in L_{\text {loc }}^{1}(\Omega)$ with $\exp \left(\mathcal{A}\left(K_{O}\right)\right) \in L_{\text {loc }}^{1}(\Omega)$, where $\mathcal{A}$ is an Orlicz function. We call a function $\mathcal{A}:[0, \infty) \rightarrow[0, \infty)$ infinitely differentiable on $(0, \infty)$ and strictly increasing with $\mathcal{A}(0)=0$ and $\lim _{t \rightarrow \infty} \mathcal{A}(t)=\infty$ an Orlicz function. We will assume for all $\Omega^{\prime} \subset \subset \Omega$ that

$$
\int_{\Omega^{\prime}} \exp \mathcal{A}\left(K_{O}(x)\right) d x<\infty
$$

where

$$
\int_{1}^{\infty} \frac{\mathcal{A}^{\prime}(s)}{s} d s=\frac{1}{\beta} \int_{0}^{\sqrt[\beta]{\frac{C}{\exp (\mathcal{A}(1))}}} \frac{d t}{t \mathcal{A}^{-1}\left(\log \frac{C}{t^{\beta}}\right)}=\infty
$$

for all $C, \beta>0$. This is condition (i) from the introduction. We wish to warn the reader that conditions (6) and (7) do not require $K_{O}$ even to be locally integrable, and thus an additional technical assumption has to be imposed on $\mathcal{A}$. To fill up this gap we assume that $\mathcal{A}$ satisfies condition (ii): $t \mathcal{A}^{\prime}(t)$ increases for large $t$ to infinity.

Proposition 2.1. Assume that $\mathcal{A}$ is an Orlicz function satisfying (i) and (ii). Then we have the pointwise inequality

$$
P(K J) \leq J+\exp (\mathcal{A}(K))-1
$$

for all $K, J \geq 0$, where the Orlicz function $P$ satisfies the integrability condition

$$
\int_{1}^{\infty} \frac{P(s)}{s^{2}}=\infty
$$

and also the technical condition that for every $\epsilon>0$, we have

$$
\left(t^{-1} P(t)\right)^{\prime} \leq 0 \leq\left(t^{\epsilon-1} P(t)\right)^{\prime}
$$

for all $t \geq 2.1(\epsilon, \mathcal{A})$.

We refer to [14 Lemma 2.1 and Lemma 2.3] for the proof of Proposition 2.1]. 
Lemma 2.2. Assume that $\mathcal{A}$ is an Orlicz function satisfying (ii), and let $p \in[1, \infty)$. Then there exists T2.2 $_{2}=t_{2.2}(p, \mathcal{A}) \in(0, \infty)$ such that the function

$$
t \rightarrow t^{-p} \exp (\mathcal{A}(t))
$$

is increasing on $(2.2, \infty)$.

Proof. The claim follows from the identity

$$
\frac{d}{d t} t^{-p} \exp (\mathcal{A}(t))=t^{-p-1} \exp (\mathcal{A}(t))\left[\mathcal{A}^{\prime}(t) t-p\right]
$$

Lemma 2.3. Assume that $\mathcal{A}$ is an Orlicz function satisfying (ii), and let $p \in[1, \infty)$. Then there exists $t_{2.3}=\frac{t 2.3}{6}(p, \mathcal{A}) \in(0, \infty)$ such that the function

$$
t \rightarrow \frac{t}{\mathcal{A}^{-1}\left(\log t^{p}\right)}
$$

is increasing on $(42.3, \infty)$.

Proof. Because

$$
\frac{d}{d t} \exp \left(\frac{\mathcal{A}(t)}{p}\right) t^{-1}=\exp \left(\frac{\mathcal{A}(t)}{p}\right) t^{-2}\left(\frac{1}{p} \mathcal{A}^{\prime}(t) t-1\right),
$$

we find a number $\tilde{t}_{0}(p, \mathcal{A})$ such that $\exp \left(\frac{\mathcal{A}(t)}{p}\right) t^{-1}$ is increasing for $t>\tilde{t}_{0}$. We conclude that

$$
\frac{t}{\mathcal{A}^{-1}\left(\log t^{p}\right)}=\frac{\exp \left(\frac{\mathcal{A}\left(\mathcal{A}^{-1}(p \log t)\right)}{p}\right)}{\mathcal{A}^{-1}(p \log t)}
$$

is increasing on $\left(t_{2.3}, \infty\right)$, where t2.3 $=\exp \left(\mathcal{A}\left(\tilde{t}_{0}\right) / p\right)$.

Lemma 2.4. Assume that $\mathcal{A}$ is an Orlicz function satisfying (ii), and let $p \in[1, \infty)$.

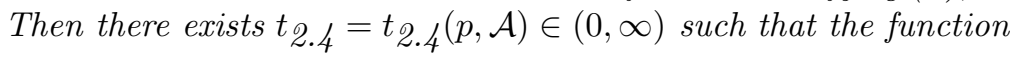

$$
t \rightarrow \exp \left(\mathcal{A}\left(t^{\frac{1}{p}}\right)\right)
$$

is convex on $(2.4, \infty)$.

Proof. Because

$$
\frac{d}{d t} \exp \left(\mathcal{A}\left(t^{\frac{1}{p}}\right)\right)=\frac{1}{p} \exp \left(\mathcal{A}\left(t^{\frac{1}{p}}\right)\right) \mathcal{A}^{\prime}\left(t^{\frac{1}{p}}\right) t^{\frac{1}{p}-1},
$$

it suffices to show that the function

$$
t \rightarrow \frac{\exp \left(\mathcal{A}\left(t^{\frac{1}{p}}\right)\right)}{t}
$$

is increasing for large values of $t$. This holds by Lemma [2.2 and so the claim follows. 


\section{ISOPERIMETRIC-TYPE INEQUALITY}

A crucial tool in establishing the correct modulus of continuity in our case is the following integral-type isoperimetric inequality for mappings in the space $\{f \in$ $W_{l o c}^{1,1}\left(\Omega, \mathbb{R}^{n}\right): P\left(|D f|^{n}\right) \in L_{l o c}^{1}(\Omega)$ and $J(x, f) \geq 0$ a.e. in $\left.\Omega\right\}$.

Lemma 3.1. Assume that an Orlicz function $P$ satisfies the divergence condition (9) and the technical condition (10). Let $f \in W_{l o c}^{1,1}\left(\Omega, \mathbb{R}^{n}\right), n \geq 2$, satisfy $P\left(|D f|^{n}\right) \in L_{l o c}^{1}(\Omega)$ with $J(x, f) \geq 0$ almost everywhere in $\Omega$. Then for each $B\left(x_{0}, R\right) \subset \subset \Omega$, we have

$$
\int_{B\left(x_{0}, r\right)} J(z, f) d z \leq\left(n \sqrt[n-1]{\omega_{n-1}}\right)^{-1}\left(\int_{\partial B\left(x_{0}, r\right)}|D f(z)|^{n-1} d z\right)^{\frac{n}{n-1}}
$$

for almost every $0<r<R$.

We refer to [17] for the proof of Lemma 3.1] Actually, Lemma 3.1] is the simple case of our more general result in [17], because we can integrate by parts against the Jacobian under the assumptions of Lemma 3.1. This means that

$$
\int_{\Omega} \varphi(x) J(x, f) d x=-\int_{\Omega} f_{i} J\left(x, f_{1}, \ldots, f_{i-1}, \varphi, f_{i+1}, \ldots, f_{n}\right) d x
$$

for all test functions $\varphi \in C_{0}^{\infty}(\Omega)$ and each index $i=1, \ldots, n$.

We close this section by giving a simple proof for a weaker version of the isoperimetric-type inequality. This is weaker in two senses. The power of $|D f|$ will be larger than $n-1$, and the constant somewhat larger. This weaker version is shown below to yield a version of Theorem 1.1 with $\frac{\lambda}{n}$ replaced by $\frac{\lambda}{n^{2} A_{p}(n)}$, where $p \in(n-1, n)$.

Lemma 3.2. Under the assumptions of Lemma 3.1, for all $p \in(n-1, n)$ we have

$$
\int_{B\left(x_{0}, r\right)} J(z, f) d z \leq \frac{A_{p}(n)}{\sqrt[n-1]{\omega_{n-1}}}\left|\partial B\left(x_{0}, r\right)\right|^{\frac{n}{n-1}}\left(f_{\partial B\left(x_{0}, r\right)}|D f(z)|^{p} d z\right)^{\frac{n}{p}}
$$

for almost every $0<r<R$. The constant $A_{p}(n)$ is given by the formula

$$
A_{p}(n)=\left(\frac{\omega_{n-1}}{\omega_{n-2}}\right)^{\frac{1}{p}}\left(\int_{0}^{\pi} \sin ^{\frac{1-n}{p-1}}(\theta) d \theta\right)^{\frac{p-1}{p}} .
$$

Because

$$
\pi \leq A_{p}(n) \leq \pi \frac{p-1}{p-n+1}
$$

(see [10, Lemma 3.10.1]), (13) is weaker than (11).

Proof. Let $j \in\{1,2,3, \ldots\}$. Choose a mollifier $\eta \in C_{0}^{\infty}(B(0,1))$ and let $\eta_{j}(x)=$ $j^{n} \eta(j x)$ and $\varphi_{j}=\eta_{j} * \chi_{B\left(x_{0}, r-1 / j\right)}$. Then $\varphi_{j} \in C_{0}^{\infty}(\Omega)$ when $j$ is sufficiently large, and $\sup \left\{\left|\nabla \varphi_{j}(x)\right|: x \in \Omega\right\} \leq j$.

Because $p>n-1$, the mapping $f$ is continuous on $\partial B\left(x_{0}, r\right)$ for almost every $r \in(0, R)$. Fix such an $r$ and pick $a \in \partial B\left(x_{0}, r\right)$. By [3] or [2, Theorem 1.1], we 
know that we can integrate by parts against the Jacobian, and so

$$
\begin{aligned}
\left|\int_{\Omega} \varphi_{j}(x) J(x, f) d x\right| & =\left|-\int_{r-\frac{1}{j}}^{r} \int_{\partial B\left(x_{0}, r\right)}\left(f_{1}(x)-f_{1}(a)\right) J\left(x, \varphi_{j}, f_{2}, \ldots, f_{n}\right) d x\right| \\
& \leq j \int_{r-\frac{1}{j}}^{r} \int_{\partial B\left(x_{0}, r\right)}\left|f_{1}(x)-f_{1}(a)\right||D f(x)|^{n-1} d x
\end{aligned}
$$

for almost every $r \in(0, R)$. Letting $j \rightarrow \infty$ and using the monotone convergence theorem and the Lebesgue differentiation theorem, we conclude that

$$
\int_{B\left(x_{0}, r\right)} J(x, f) d x \leq \operatorname{osc}\left(f_{1}, \partial B\left(x_{0}, r\right)\right) \int_{\partial B\left(x_{0}, r\right)}|D f(x)|^{n-1} d x
$$

for almost every $r \in(0, R)$. Here we used the standard notation

$$
\operatorname{osc}\left(f_{1}, \partial B\left(x_{0}, r\right)\right)=\sup _{\partial B\left(x_{0}, r\right)} f_{1}(x)-\inf _{\partial B\left(x_{0}, r\right)} f_{1}(x) .
$$

The oscillation lemma ([10, Lemma 3.10.1]) tells us that

$$
\operatorname{osc}\left(f_{1}, \partial B\left(x_{0}, r\right)\right) \leq A_{p}(n) r\left(f_{\partial B\left(x_{0}, r\right)}\left|\nabla f_{1}\right|^{p}\right)^{\frac{1}{p}},
$$

where the constant $A_{p}(n)$ is given by the formula (13). Combining the inequality (16) with the oscillation lemma and using Hölder's inequality, we conclude that

$$
\int_{B\left(x_{0}, r\right)} J(z, f) d z \leq \frac{A_{p}(n)}{\sqrt[n-1]{\omega_{n-1}}}\left|\partial B\left(x_{0}, r\right)\right|^{\frac{n}{n-1}}\left(f_{\partial B\left(x_{0}, r\right)}|D f(z)|^{p} d z\right)^{\frac{n}{p}}
$$

for almost every $0<r<R$, as desired.

\section{Proof of Theorem 1.1}

The elements in the Sobolev space $W_{l o c}^{1,1}(\Omega)$ are equivalence classes of functions that agree almost everywhere in $\Omega$. In order to study the fine properties of a function $u \in W_{l o c}^{1,1}(\Omega)$, it is convenient to use the representative $\tilde{u}$, defined by the formula

$$
\tilde{u}(x)=\lim _{r \rightarrow 0} \sup f_{B(x, r)} u(z) d z .
$$

If $f \in W_{l o c}^{1,1}\left(\Omega, \mathbb{R}^{n}\right)$, then we set $\tilde{f}=\left(\tilde{f}_{1}, \ldots, \tilde{f}_{n}\right)$. It is well known that if $v: \mathbb{R}^{n} \rightarrow \mathbb{R}$ is locally integrable, then

$$
\lim _{r \rightarrow 0} f_{B(x, r)}|v(x)-v(z)| d z=0
$$

for almost all $x \in \mathbb{R}^{n}$, and thus $\tilde{f}=f$ almost everywhere in $\Omega$.

Let $\mathcal{A}$ be an Orlicz function satisfying the integrability condition (7), $n \in$ $\{2,3,4, \ldots\}, K>0$ and $\beta>0$. We introduce the strictly increasing function $\alpha(r)=\alpha_{\mathcal{A}, K, n, \beta}(r)$ defined for $0<r^{n}<\frac{n K}{\omega_{n-1}}$ by the formula

$$
\alpha_{\mathcal{A}, K, n, \beta}(r)=\sup \left\{t \in\left(0, \frac{r}{2}\right): \int_{t}^{r / 2} \frac{d s}{s \mathcal{A}^{-1}\left(\log \frac{n K}{\omega_{n-1} s^{n}}\right)} d s \geq \beta\right\} .
$$


Now we can formulate our main theorem. After stating the result, we show that this technical version easily yields Theorem 1.1, for a slightly simpler version, see Remark 4.4 below.

Theorem 4.1. Assume that an Orlicz function $\mathcal{A}$ satisfies (i) and (ii). Let $f$ : $\Omega \rightarrow \mathbb{R}^{n}$ be a mapping of finite distortion whose distortion function satisfies the integrability condition

$$
K=\int_{B} \exp \left(\mathcal{A}\left(K_{O}(x)\right)\right) d x<\infty,
$$

where $B=B\left(x_{0}, R\right) \subset \subset \Omega$. Then, for all small $\epsilon>0$, we have

$$
\begin{aligned}
|\tilde{f}(x)-\tilde{f}(y)| \leq & C_{\mathcal{A}, K}(n, \beta) \exp \left((\epsilon-1) \int_{2 \alpha^{-1}(|x-y|)}^{R} \frac{d t}{t \mathcal{A}^{-1}\left(\log C_{\mathcal{A}, n}(\epsilon) \frac{n K}{\omega_{n-1} t^{n}}\right)}\right) \\
& \times\left(\int_{B} J(z, f) d z\right)^{\frac{1}{n}}
\end{aligned}
$$

whenever $x, y \in B\left(x_{0}, \alpha(R / 4)\right)$.

We will split the proof of Theorem 4.1 into two parts, Lemma 4.2 and Lemma 4.3. Before we go to the proof of Theorem 4.1 let us show how Theorem 1.1 follows from Theorem 4.1; Let $\mathcal{A}(t)=\lambda t, \lambda>0$ and $s(r)=\left(\frac{r}{2}\right)^{e}\left[\frac{n K}{\omega_{n-1}}\right]^{\frac{1-e}{n}}$. First we observe that

$$
\int_{s(r)}^{r / 2} \frac{d t}{t \mathcal{A}^{-1}\left(\log \frac{n K}{\omega_{n-1} t^{n}}\right)}=\frac{\lambda}{n}
$$

and so $\alpha_{\mathcal{A}, K, n, \lambda / n}(r) \geq s(r)$ for all $r<\sqrt[n]{\frac{n K}{\omega_{n-1}}}$. Furthermore,

$$
\begin{gathered}
\exp \left((\epsilon-1) \int_{2 s^{-1}(|x-y|)}^{R} \frac{d t}{t \mathcal{A}^{-1}\left(\log C_{\mathcal{A}, n}(\epsilon) \frac{n K}{\omega_{n-1} t^{n}}\right)}\right) \\
=\left(\frac{\log \left(C_{\mathcal{A}, n}(\epsilon) \frac{n K}{\omega_{n-1} R^{n}}\right)}{\log \left(\frac{C_{\mathcal{A}, n}(\epsilon)}{2^{n+\frac{n}{e}}}\left[\frac{n K}{\omega_{n-1}|x-y|^{n}}\right]^{\frac{1}{e}}\right)}\right)^{\frac{\lambda}{n}(1-\epsilon)},
\end{gathered}
$$

and so Theorem 1.1 follows.

Lemma 4.2. Under the hypothesis of Theorem 4.1. we have

$$
|\tilde{f}(x)-\tilde{f}(y)|^{n} \int_{r}^{R / 2} \frac{d t}{t \mathcal{A}^{-1}\left(\log \frac{n K}{\omega_{n-1} t^{n}}\right)} \leq C_{\mathcal{A}, K}(n) \int_{B\left(x_{0}, R\right)} J(z, f) d z
$$

whenever $x, y \in B\left(x_{0}, r\right) \subset B\left(x_{0}, R / 2\right)$.

Proof. Combining the distortion inequality $|D f(x)|^{n} \leq K_{O}(x) J(x, f)$ with Proposition 2.1, we obtain that $P\left(|D f|^{n}\right) \in L_{l o c}^{1}(\Omega)$, where $\int_{1}^{\infty} \frac{P(s)}{s^{2}}=\infty$ and for every $\epsilon>0$, the function $t \rightarrow t^{\epsilon-1} P(t)$ is increasing for all $t \geq 2.1(\mathcal{A}, \epsilon)$. Using Proposition 2.6 in [14] and Proposition 2.4 in [14, we conclude that the coordinate functions of $f$ are weakly monotone (see [7, Definition 1.5]). This is based on the fact that $J(\cdot, f)$ coincides with the distributional Jacobian, i.e., (12) holds. Let $p=n-\frac{1}{2}$, $r<R / 2$ and $x, y \in B\left(x_{0}, r\right)$. Then $|D f| \in L_{l o c}^{p}(\Omega)$. Using Lemma 7.2 in [7], which 
holds for mappings whose coordinate functions are weakly monotone, we have the estimate

$$
\frac{|\tilde{f}(x)-\tilde{f}(y)|}{C(n, p) t} \leq\left(f_{\partial B\left(x_{0}, t\right)}|D f|^{p}\right)^{\frac{1}{p}}
$$

for almost every $t \in[r, R]$. Write $B_{s}=B\left(x_{0}, s\right)$ and $A_{i}=B_{2^{i} r} \backslash B_{2^{i-1} r}$, for all $i \in\{1,2, \ldots\}$. Define

$$
G_{i}=\left\{t \in\left[2^{i-1} r, 2^{i} r\right]: \int_{\partial B_{t}} \exp \left(\mathcal{A}\left(K_{O}(x)\right)\right) d x \leq \frac{3}{2^{i-1} r} \int_{A_{i}} \exp \left(\mathcal{A}\left(K_{O}(x)\right)\right) d x\right\}
$$

for all $i \in\{1,2, \ldots\} \cap\left[1, \log _{2} \frac{R}{r}\right]=I$. Because $2 r \leq R$, we see that $I \neq \emptyset$. Using Fubini's theorem, we conclude that

$$
\left|G_{i}\right| \geq \frac{2^{i-1}}{2} r
$$

for all $i \in I$. Combining the distortion inequality and Hölder's inequality with the inequality (23), we have that

$$
\frac{|\tilde{f}(x)-\tilde{f}(y)|^{n}}{C(n, p) t^{n}} \leq\left(f_{\partial B_{t}}\left|K_{O}(x)\right|^{\frac{p}{n-p}}\right)^{\frac{n-p}{p}} f_{\partial B_{t}} J(x, f) d x
$$

for almost every $t \in[r, R]$. By Lemma 2.2. we find a number $\llbracket 2.2\left(\frac{p}{n-p}, \mathcal{A}\right)$ such that the function $t \rightarrow t^{-\frac{p}{n-p}} \exp \mathcal{A}(t)$ is increasing on $(42.2, \infty)$. Let $t \in[r, R]$ be such that $f_{\partial B_{t}} \exp \left(\mathcal{A}\left(K_{O}(x)\right)\right) d x<\infty$, and pick $\tau$ so that

$$
\exp \mathcal{A}(\tau)=f_{\partial B_{t}} \exp \left(\mathcal{A}\left(K_{O}(x)\right)\right) d x
$$

Write $\lambda=\max \{\tau, \not 2.2\}$. Then we estimate

$$
\begin{aligned}
f_{\partial B_{t}}\left|K_{O}(x)\right|^{\frac{p}{n-p}} d x \leq & \frac{1}{\left|\partial B_{t}\right|} \int_{\partial B_{t} \cap\left\{\left|K_{O}\right|>\lambda\right\}}\left|K_{O}(x)\right|^{\frac{p}{n-p}} d x \\
& +\int_{\partial B_{t} \cap\left\{\left|K_{O}\right| \leq \lambda\right\}}\left|K_{O}(x)\right|^{\frac{p}{n-p}} d x \\
\leq & \frac{\lambda^{\frac{p}{n-p}}}{\mathcal{A}(\lambda)} \int_{\partial B_{t}} \exp \left(\mathcal{A}\left(K_{O}(x)\right)\right) d x+\lambda^{\frac{p}{n-p}} \leq 2 \lambda^{\frac{p}{n-p}}
\end{aligned}
$$

It follows that

$$
\frac{|\tilde{f}(x)-\tilde{f}(y)|^{n}}{t} \leq C(p, n) \lambda \int_{\partial B_{t}} J(x, f) d x
$$

and thus

$$
\begin{aligned}
\frac{|\tilde{f}(x)-\tilde{f}(y)|^{n}}{t} \leq & C(p, n, \mathcal{A}) \mathcal{A}^{-1}\left(\log \left(f_{\partial B_{t}} \exp \left(\mathcal{A}\left(K_{O}(x)\right)\right) d x\right)\right) \\
& \times \int_{\partial B_{t}} J(x, f) d x
\end{aligned}
$$

for almost every $t \in[r, R]$. Fix $i \in I$. For almost every $t \in G_{i}$, we have that

$$
\frac{|\tilde{f}(x)-\tilde{f}(y)|^{n}}{t} \leq C(p, n, \mathcal{A}) \mathcal{A}^{-1}\left(\log \left(\frac{6 K}{\omega_{n-1} t^{n-1} 2^{i} r}\right)\right) \int_{\partial B_{t}} J(x, f) d x .
$$


Integrating this estimate over the set $G_{i}$ with respect to $t$, we arrive at

$$
|\tilde{f}(x)-\tilde{f}(y)|^{n} \int_{G_{i}} \frac{d t}{t \mathcal{A}^{-1}\left(\log \frac{6 n K}{\omega_{n-1} t^{n}}\right)} \leq C(p, n, \mathcal{A}) \int_{A_{i}} J(x, f) d x .
$$

By Lemma 2.3 we fix $2.3=42.3(n, \mathcal{A}) \geq 1$ so that the function $h: t \rightarrow$ $\left[t \mathcal{A}^{-1}\left(\log \left(\frac{1}{t^{n}}\right)\right)\right]^{-1}$ is decreasing on $\left(0, \frac{1}{2.3}\right)$. Then $t \rightarrow h\left(\frac{t}{2.3} \sqrt[n]{\frac{\omega_{n-1}}{6 n K}}\right)$ is decreasing on $\left(0, \sqrt[n]{\frac{6 n K}{\omega_{n-1}}}\right) \supset(0, R)$. Combining this with the estimate $\left|G_{i}\right| \geq \frac{2^{i-1}}{2} r$, we conclude that

$$
|\tilde{f}(x)-\tilde{f}(y)|^{n} \int_{2^{i-2} 3 r}^{2^{i} r} \frac{d t}{t \mathcal{A}^{-1}\left(\log \frac{\frac{t^{n}}{\omega_{n-1} t^{n}}}{\omega^{K}}\right)} \leq C(p, n, \mathcal{A}) \int_{A_{i}} J(x, f) d x .
$$

Because

$$
\begin{aligned}
\int_{2^{i-2} 3 r}^{2^{i} r} \frac{d t}{t \mathcal{A}^{-1}\left(\log \frac{\left|\frac{n}{2.3}\right|_{n-1} t^{n}}{\omega^{n}}\right)} & =\int_{2^{i-1} r}^{2^{i-2} 3 r} \frac{d s}{\left(s+2^{i-2} r\right) \mathcal{A}^{-1}\left(\log \left(\frac{\frac{1}{2.3}^{n K}}{\omega_{n-1}\left(s+2^{i-2} r\right)^{n}}\right)\right)} \\
& \geq \int_{2^{i-1} r}^{2^{i-2} 3 r} \frac{1}{3} \frac{d s}{s \mathcal{A}^{-1}\left(\log \frac{\mid \frac{\mid n}{\omega_{n-1} .3 s^{n}}}{\omega^{n}}\right)},
\end{aligned}
$$

we obtain the estimate

$$
|\tilde{f}(x)-\tilde{f}(y)|^{n} \int_{2^{i-1} r}^{2^{i} r} \frac{d t}{t \mathcal{A}^{-1}\left(\log \frac{\frac{t^{n}}{\omega_{n-1} t^{n}}}{\omega_{n}}\right)} \leq C(p, n, \mathcal{A}) \int_{A_{i}} J(x, f) d x .
$$

Summing over the set $I$, we arrive at

$$
|\tilde{f}(x)-\tilde{f}(y)|^{n} \int_{r}^{R / 2} \frac{d t}{t \mathcal{A}^{-1}\left(\log \frac{t^{n} \frac{n}{\omega_{n-1} t^{n}}}{\omega^{n}}\right)} \leq C(p, n, \mathcal{A}) \int_{B_{R}} J(x, f) d x .
$$

In the case $t \in\left(0, \sqrt[n]{\frac{n K}{\omega_{n-1}[\sqrt{2.3}}}\right)$, we have that

$$
\begin{aligned}
& \frac{1}{t \mathcal{A}^{-1}\left(\log \frac{\frac{t n}{2.33^{K}}}{t^{n}}\right)}=\frac{1}{\sqrt{2.3}} \frac{\sqrt{2.3}}{t \mathcal{A}^{-1}\left(\log \frac{\frac{t n}{2.3}}{\omega_{n-1} t^{n}}\right)} \\
& \geq \frac{1}{42.3} \frac{1}{t \mathcal{A}^{-1}\left(\log \frac{n K}{\omega_{n-1} t^{n}}\right)} .
\end{aligned}
$$

Here we used the fact that the function $s \rightarrow \frac{s}{\mathcal{A}^{-1}\left(\log s^{n}\right)}$ is increasing on $\left(t_{2.3}, \infty\right)$. Furthermore,

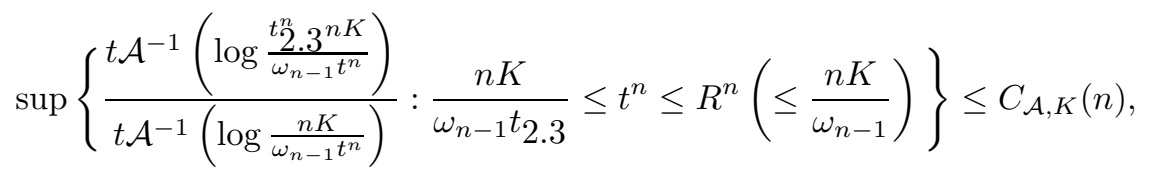


and so also in the case $R^{n} \geq t^{n} \geq \frac{n K}{\omega_{n-1} \sqrt{2.3}}$ we have the inequality

$$
t \mathcal{A}^{-1}\left(\log \frac{n K}{\omega_{n-1} t^{n}}\right) \geq C_{\mathcal{A}, K}(n) t \mathcal{A}^{-1}\left(\log \frac{\frac{\frac{n}{2.3}}{\omega_{n-1} t^{n}}}{\omega_{n}} .\right.
$$

Combining the inequality (32) with the estimates (33) and (35), we complete the proof of Lemma 4.2.

Inequality (22) together with the following lemma gives us the desired modulus of continuity.

Lemma 4.3. Under the hypothesis of Theorem 4.1, for every small $\epsilon>0$, we have

$$
\begin{aligned}
\int_{B\left(x_{0}, r\right)} J(x, f) d x \leq & 2 \exp \left((\epsilon-n) \int_{2 r}^{R} \frac{d t}{t \mathcal{A}^{-1}\left(\log C_{\mathcal{A}, n}(\epsilon) \frac{n K}{\omega_{n-1} t^{n}}\right)}\right) \\
& \times \int_{B\left(x_{0}, R\right)} J(x, f) d x
\end{aligned}
$$

whenever $r \in(0, R / 2)$.

Proof. Using Proposition 2.1, we see that the assumptions of Lemma 3.1 are fulfilled, and so

$$
\int_{B\left(x_{0}, s\right)} J(x, f) d x \leq\left(n \sqrt[n-1]{\omega_{n-1}}\right)^{-1}\left|\partial B\left(x_{0}, r\right)\right|^{\frac{n}{n-1}}\left(f_{\partial B(x, s)}|D f|^{p}\right)^{\frac{n}{p}}
$$

for almost every $0<s<R$ and all $p \geq n-1$. To get the sharp estimate, we will later take $p=n-1$; the general value of $p$ allows us to show that already Lemma 3.2 is sufficient to establish a logarithmic modulus of continuity. Write $B_{r}=B\left(x_{0}, r\right)$. Fix $\epsilon \in(0,1), p \in[n-1, n)$, and $i \in\{1,2,3, \ldots\}$. We denote the interval $\left(R 2^{-\epsilon i}, R 2^{-\epsilon(i-1)}\right)$ by $\triangle_{i, \epsilon}$ and the annulus $B_{R 2^{-\epsilon(i-1)}} \backslash B_{R 2^{-\epsilon i}}$ by $A_{i, \epsilon}$. Using Fubini's theorem, we observe that for every $\delta>0$, the set

$$
E_{i}=\left\{t \in \triangle_{i, \epsilon}: \int_{\partial B_{t}}|D f|^{p} \leq \frac{(1+\delta)}{\left|\triangle_{i, \epsilon}\right|} \int_{A_{i, \epsilon}}|D f(x)|^{p} d x\right\}
$$

has a positive measure.

Choosing $r \in \triangle_{i, \epsilon}$ so that $r$ lies in the set $E_{i}$ and so that the inequality (37) holds, we obtain the estimate

$$
\begin{aligned}
\int_{B_{R 2}-\epsilon i} J(x, f) d x \leq & \int_{B_{r}} J(x, f) d x \leq\left(\sqrt[n-1]{\omega_{n-1}}\right)^{-1}\left|\partial B_{r}\right|^{\frac{n}{n-1}}\left(\int_{\partial B_{r}}|D f|^{p}\right)^{\frac{n}{p}} \\
\leq & \left(n \sqrt[n-1]{\omega_{n-1}}\right)^{-1}\left(\frac{1+\delta}{\left|\triangle_{i, \epsilon}\right|}\right)^{\frac{n}{p}}\left|\partial B_{r}\right|^{\frac{n}{n-1}-\frac{n}{p}} \\
& \times\left(\int_{A_{i, \epsilon}}|D f(x)|^{p} d x\right)^{\frac{n}{n-1}}
\end{aligned}
$$


By Lemma 2.4, we can fix t2.4] $=42.4(p /(n-p), \mathcal{A}) \in(0, \infty)$ so that the function $t \rightarrow \exp \mathcal{A}\left(t^{\frac{n-p}{p}}\right)$ is convex on $(2.4, \infty)$. We set

$$
\tilde{K}_{O}(x)= \begin{cases}K_{O}(x), & K_{O}(x)>2.4 \\ 2.4 & K_{O}(x) \leq 2.4\end{cases}
$$

Combining the distortion inequality $|D f(x)|^{n} \leq \tilde{K}_{O}(x) J(x, f)$ with Hölder's inequality, we find that

$$
\begin{aligned}
& \int_{B_{R 2}-\epsilon i} J(x, f) d x \leq\left(n \sqrt[n-1]{\omega_{n-1}}\right)^{-1}\left(\frac{1+\delta}{\left|\triangle_{i, \epsilon}\right|}\right)^{\frac{n}{p}}\left|\partial B_{r}\right|^{\frac{n}{n-1}-\frac{n}{p}} \\
& \times\left(\int_{A_{i, \epsilon}}\left|\tilde{K}_{O}\right|^{\frac{p}{n-p}}\right)^{\frac{n-p}{p}} \int_{A_{i, \epsilon}} J(x, f) d x
\end{aligned}
$$

Jensen's inequality applied to the convex function $(42.4, \infty) \rightarrow(0, \infty): \tau \rightarrow$ $\exp \mathcal{A}\left(\tau^{\frac{n-p}{p}}\right)$ yields

$$
\begin{aligned}
\int_{B_{R 2}-\epsilon i} J(x, f) d x \leq & \left(n \sqrt[n-1]{\omega_{n-1}}\right)^{-1}\left(\frac{1+\delta}{\left|\triangle_{i, \epsilon}\right|}\right)^{\frac{n}{p}}\left|A_{i, \epsilon}\right|^{\frac{n-p}{p}} \\
\times & \times\left.\partial B_{r}\right|^{\frac{n}{n-1}-\frac{n}{p}} \mathcal{A}^{-1}\left(\log f_{A_{i, \epsilon}} \exp \mathcal{A}\left(\tilde{K}_{O}\right)\right) \int_{A_{i, \epsilon}} J(x, f) d x
\end{aligned}
$$

and computations show that

$$
\begin{aligned}
\int_{B_{R 2}-\epsilon i} J(x, f) d x \leq & \left(\frac{1+\delta}{n}\right)^{\frac{n}{p}}\left(\frac{2^{\epsilon n}-1}{2^{\epsilon}-1}\right)^{\frac{n-p}{p}} R 2^{-\epsilon i}\left|\triangle_{i, \epsilon}\right|^{-1} \\
& \times \mathcal{A}^{-1}\left(\log \left(\frac{\exp \mathcal{A}\left(t_{2.4}\right) n K 2^{\epsilon n}}{\omega_{n-1} R^{n} 2^{-\epsilon n(i-1)}\left(2^{\epsilon n}-1\right)}\right)\right) \\
& \times \int_{A_{i, \epsilon}} J(x, f) d x .
\end{aligned}
$$

Letting $\delta \rightarrow 0$, using the elementary inequalities $\epsilon a \log 2 \leq 2^{a \epsilon}-1 \leq \epsilon a 2^{a \epsilon} \log 2$ for all $\epsilon, a \geq 0$ and the fact that $\epsilon \leq 1$, we conclude that

$$
\begin{aligned}
\int_{B_{R 2}-\epsilon i} J(x, f) d x \leq & \frac{2^{\frac{n \epsilon}{p}}}{n} R 2^{-\epsilon i} \mathcal{A}^{-1}\left(\log \frac{\tilde{C} n K}{\omega_{n-1} R^{n} 2^{-\epsilon n(i-1)} \epsilon}\right) \\
& \times \int_{A_{i, \epsilon}} J(x, f) d x\left|\triangle_{i, \epsilon}\right|^{-1} .
\end{aligned}
$$

Here and also in what follows, we denote the constant $\exp \mathcal{A}(\overline{2.4}(p /(n-p), \mathcal{A}))$ by $\tilde{C}$. At this point we would like to warn the reader that the constant $\tilde{C}$ tends to infinity if we let $p \rightarrow n$.

Next we introduce two auxiliary functions. We set

$$
u_{i, \epsilon}(x)=\int_{B_{R 2}-\epsilon i} J(x, f) d x+\frac{x-R 2^{-\epsilon i}}{\left|\triangle_{i, \epsilon}\right|} \int_{A_{i, \epsilon}} J(x, f) d x
$$


and

$$
u_{\epsilon}=\sum_{i=1}^{\infty} u_{i, \epsilon}(x) \chi_{\left[R 2^{-\epsilon i}, R 2^{-\epsilon(i-1)}\right)}(x)
$$

for every $x \in \mathbb{R}$. Using the inequality (42), we see that

$$
u_{i, \epsilon}(x) \leq\left[\frac{2^{\frac{n \epsilon}{p}}}{n} R 2^{-\epsilon i} \mathcal{A}^{-1}\left(\log \frac{\tilde{C} n K}{\omega_{n-1} R^{n} 2^{-\epsilon n(i-1)} \epsilon}\right)+x-R 2^{-\epsilon i}\right] u_{i, \epsilon}^{\prime}(x)
$$

for all $x \in \triangle_{i, \epsilon}$. Combining the inequality $R 2^{-\epsilon(i-1)}-R 2^{-\epsilon i} \leq R 2^{-\epsilon i} 2 \epsilon$ with the fact that

$$
\left[\mathcal{A}^{-1}\left(\log \frac{\tilde{C} n K}{\omega_{n-1} R^{n} 2^{-\epsilon n(i-1)} \epsilon}\right)\right]^{-1} \leq\left[\mathcal{A}^{-1}\left(\log \frac{2^{\epsilon n} \tilde{C} n K}{\epsilon \omega_{n-1} R^{n}}\right)\right]^{-1}=B_{\epsilon}
$$

(notice that $B_{\epsilon} \rightarrow 0$ as $\epsilon \rightarrow 0$ ), we conclude with

$$
u_{i, \epsilon}(x) \leq\left(\frac{2^{\frac{n \epsilon}{p}}}{n}+2 B_{\epsilon} \epsilon\right) x \mathcal{A}^{-1}\left(\log \frac{\tilde{C} n K}{\omega_{n-1} x^{n} \epsilon}\right) u_{i, \epsilon}^{\prime}(x)
$$

for all $x \in \triangle_{i, \epsilon}$.

Fix $\delta \in\left(0, R / 10^{6}\right)$ and define

$$
v_{\epsilon, \delta}(x)=\exp \left(-\int_{\delta}^{x}\left[\left(\frac{2^{\frac{n \epsilon}{p}}}{n}+2 B_{\epsilon} \epsilon\right) t \mathcal{A}^{-1}\left(\log \frac{\tilde{C} n K}{\omega_{n-1} t^{n} \epsilon}\right)\right]^{-1} d t\right)
$$

for all $x \in(\delta, R)$. Using the inequality (44), we see that

$$
\begin{aligned}
\frac{d}{d x}\left(v_{\epsilon, \delta} u_{\epsilon}\right)(x)=v_{\epsilon, \delta}(x)\left[-\left(\left(\frac{2^{\frac{n \epsilon}{p}}}{n}+2 B_{\epsilon} \epsilon\right) x \mathcal{A}^{-1}\left(\log \frac{\tilde{C} n K}{\omega_{n-1} x^{n} \epsilon}\right)\right)^{-1}\right. \\
\left.u_{\epsilon}(x)+u_{\epsilon}^{\prime}(x)\right] \geq 0
\end{aligned}
$$

for all $x \in(\delta, R) \cap \bigcup_{i=1}^{\infty} \triangle_{i, \epsilon}$, and so the continuous function $v_{\epsilon, \delta} u_{\epsilon}$ is nondecreasing on $(\delta, R)$. Let $2 r \in(\delta, R-\delta)$. Choosing $i_{r} \in \mathbb{N}$ so that $r \in\left[R 2^{-\epsilon i_{r}}, R 2^{-\epsilon\left(i_{r}-1\right)}\right)$, we find that

$$
v_{\epsilon, \delta}(r) \int_{B_{R 2}-\epsilon i_{r}} J(x, f) d x \leq 2 v_{\epsilon, \delta}(R-\delta) \int_{B_{R-\delta}} J(x, f) d x .
$$

Since the Jacobian of $f$ is nonnegative almost everywhere, this yields the estimate

$$
\begin{aligned}
\int_{B_{2-\epsilon_{2 r}}} J(x, f) d x \leq & 2 \exp \left[-\int_{2 r}^{R-\delta}\left[\left(\frac{2^{\frac{n \epsilon}{p}}}{n}+2 B_{\epsilon} \epsilon\right) t\right.\right. \\
& \left.\left.\times \mathcal{A}^{-1}\left(\log \frac{\tilde{C} n K}{\omega_{n-1} t^{n} \epsilon}\right)\right]^{-1} d t\right] \int_{B_{R-\delta}} J(x, f) d x .
\end{aligned}
$$

Letting $\delta \rightarrow 0$ and using the estimate $\epsilon \leq 1$, we find that

$$
\begin{aligned}
\int_{B_{r}} J(x, f) d x \leq & 2 \exp \left[-\int_{2 r}^{R}\left[\left(\frac{2^{\frac{n \epsilon}{p}}}{n}+2 B_{\epsilon} \epsilon\right) t\right.\right. \\
& \left.\left.\times \mathcal{A}^{-1}\left(\log \frac{\tilde{C} n K}{\omega_{n-1} t^{n} \epsilon}\right)\right]^{-1} d t\right] \int_{B_{R}} J(x, f) d x
\end{aligned}
$$


for all $0<r<R / 2$. Choosing $p=n-1$, the constant $\tilde{C}$ depends only on $\mathcal{A}$ and $n$. Furthermore, using the fact that $\frac{2^{\frac{n \epsilon}{n-1}}}{n}+2 B_{\epsilon} \epsilon \rightarrow \frac{1}{n}$ as $\epsilon \rightarrow 0$, we obtain the desired inequality 36 .

Now we complete the proof of the entire theorem as follows.

Given $x \in B\left(x_{0}, \alpha\left(\frac{R}{4}\right)\right)$, we consider the ball $B\left(x_{0}, r\right), r=\alpha_{\mathcal{A}, n, \beta}^{-1}\left(\left|x-x_{0}\right|\right)$. By Lemma 4.2, we have the estimate

$$
\left|\tilde{f}(x)-\tilde{f}\left(x_{0}\right)\right| \leq C_{\mathcal{A}, K}(n, \beta)\left(\int_{B\left(x_{0}, r\right)} J(z, f) d z\right)^{\frac{1}{n}} .
$$

Using Lemma 4.3, we further obtain the estimate

$$
\begin{aligned}
\int_{B\left(x_{0}, r\right)} J(z, f) d z \leq & 2 \exp \left((\epsilon-n) \int_{2 r}^{R} \frac{d t}{t \mathcal{A}^{-1}\left(\log C_{\mathcal{A}, n}(\epsilon) \frac{n K}{\omega_{n-1} t^{n}}\right)}\right) \\
& \times \int_{B\left(x_{0}, R\right)} J(x, f) d x .
\end{aligned}
$$

Combining the inequality (50) with the estimate (51), we finally obtain the desired modulus of continuity 21).

Remark 4.4. The integral in (21) of Theorem 4.1 can be taken from $|x-y|$ to $R$ when $|x-y|$ is sufficiently small. To see this, notice that the ratio of this integral, taken from $|x-y|$ to $\alpha^{-1}(|x-y|)$, with the corresponding integral from $|x-y|$ to $R$, tends to zero when $|x-y|$ tends to zero. Thus the small error made in changing the limits can be imbedded in the estimate by changing the $\epsilon$ in front of the integral in (21) to $2 \epsilon$. We leave the details to the reader.

Remark 4.5. If we use the inequality (13) instead of the isoperimetric inequality (11) in the proof of Lemma 4.3, then under the assumptions of Lemma 4.3, for every small $\epsilon>0$, we have

$$
\begin{aligned}
\int_{B\left(x_{0}, r\right)} J(x, f) d x \leq & 2 \exp \left(\left(\epsilon-\frac{1}{A_{n-\epsilon}(n)}\right) \int_{2 r}^{R} \frac{d t}{t \mathcal{A}^{-1}\left(\log C_{\mathcal{A}, n}(\epsilon) \frac{n K}{\omega_{n-1} t^{n}}\right)}\right) \\
& \times \int_{B\left(x_{0}, R\right)} J(x, f) d x
\end{aligned}
$$

whenever $r \in(0, R / 2)$. The constant $A_{n-\epsilon}(n)$ is given by the formula (14). This results in the claim of Theorem 4.1 with $\epsilon-1$ replaced by $\epsilon-\frac{1}{n A_{n-\epsilon}(n)}$, which is weaker than our claim. However, in the setting of Theorem 1.1 this weaker estimate still gives us a logarithmic modulus of continuity, this time with the exponent $\frac{\lambda}{n^{2} A_{n-\epsilon}(n)}-\epsilon$.

\section{Construction of Example 1.2}

We close this paper by showing that the modulus of continuity that we gave above in Section 4 cannot be substantially improved on. To see that Example 5.1 qualifies for that purpose, make a change of variables to reduce the integrand to the form given in Theorem 4.1, and notice that the role of $\alpha^{-1}$ is not significant when $|x-y|$ is small; see Remark 4.4. Example 1.2 is contained as a special case in Example 5.1. 
Example 5.1. Assume that an Orlicz function $\mathcal{A}$ satisfies (i). Then there exist a continuous mapping of finite distortion $f: B(0,1) \rightarrow \mathbb{R}^{n}$ such that

$$
\int_{B(0,1)} \exp \left(\mathcal{A}\left(K_{O}(x, f)\right)\right) d x<\infty
$$

and points $x_{j}, y_{j} \in B(0,1)$ so that $x_{j} \rightarrow 0, y_{j} \rightarrow 0$, and

$$
\left|f\left(x_{j}\right)-f\left(y_{j}\right)\right| \exp \left(\int_{\left|x_{j}-y_{j}\right|}^{1 / 2} \frac{d s}{s \mathcal{A}^{-1}\left(\frac{n}{1+\epsilon} \log \frac{1}{s}\right)}\right) \rightarrow \infty
$$

for every $\epsilon>0$.

Let $r \in(0,1)$. Given a ball $B=\left\{x \in \mathbb{R}^{n}:|x-a|<r\right\}$ and $\epsilon>0$, we consider the radial stretching

$$
g(x)=a+r \frac{x-a}{|x-a|} \rho_{r}(|x-a|),
$$

where

$$
\rho_{r}(t)=\exp \left(-\int_{t}^{r} \frac{d s}{s \mathcal{A}^{-1}\left(\frac{n}{1+\epsilon} \log \frac{1}{s}\right)}\right)
$$

for all $t \in(0, r)$. We may calculate the Jacobian of $g$ by using the familiar formula

$$
J(x, g)=r^{n} \rho_{r}^{\prime}(|x-a|)\left(\frac{\rho_{r}(|x-a|)}{|x-a|}\right)^{n-1}
$$

(see the equations (5.43) and (5.44) in [10]). The distortion function is

$$
K_{O}(x, g)=\frac{|D g(x)|^{n}}{J(x, g)}=\frac{\rho_{r}(|x-a|)}{|x-a| \rho_{r}^{\prime}(|x-a|)}
$$

(see the formula (5.49) in [10]). Here we used the assumption $|x-a|<1$. Using polar coordinates, we compute the integral of the Jacobian as

$$
\int_{B} J(x, g) d x=\frac{\omega_{n-1}}{n} r^{n} \int_{0}^{r} \rho_{r}^{n-1}(t) \rho_{r}^{\prime}(t) d t=\left.\frac{\omega_{n-1}}{n} r^{n} \rho_{r}^{n}(t)\right|_{0} ^{r}=|B| .
$$

Combining the equation (57) with the fact that

$$
\frac{t \rho_{r}^{\prime}(t)}{\rho_{r}(t)}=\frac{1}{\mathcal{A}^{-1}\left(\frac{n}{1+\epsilon} \log \frac{1}{t}\right)}
$$

we see that

$$
\int_{B} \exp \left(\mathcal{A}\left(K_{O}(x, g)\right)\right) d x=\int_{B}\left(\frac{1}{|x|}\right)^{\frac{n}{1+\epsilon}} d x .
$$

We will now glue together several versions of $g$ with variable $\epsilon, r$, and $a$. Let $\epsilon_{i}=\frac{1}{i}$, $i=2,3, \ldots$ For all $i=2,3,4 \ldots$ we choose $r_{i} \in\left(0,2^{-i}\right]$ so that $\int_{B\left(0, r_{i}\right)}|x|^{-\frac{n}{1+\epsilon_{i}}} d x \leq$ $2^{-i}$. Let $a_{i}=\left(2^{-i+2}, 0, \ldots, 0\right)$ for $i \geq 4$, set $B_{i}=B\left(a_{i}, r_{i}\right)$, and define $G=\bigcup_{i \geq 4} B_{i}$.

Define the mapping $f: B(0,1) \rightarrow \mathbb{R}^{n}$ by setting

$$
f(x)= \begin{cases}a_{i}+r_{i} \frac{x-a_{i}}{\left|x-a_{i}\right|} \rho_{r_{i}}\left(\left|x-a_{i}\right|\right) & \text { for } x \in B_{i}, \\ x & \text { for } x \in B(0,1) \backslash G .\end{cases}
$$


On account of the formula (59), we find that

$$
\int_{G} \exp \left(\mathcal{A}\left(K_{O}(x, f)\right)\right) \leq \sum_{i=2}^{\infty} 2^{-i}<\infty .
$$

We also have the trivial estimate

$$
\int_{B(0,1) \backslash G} \exp \left(\mathcal{A}\left(K_{O}(x, f)\right)\right) \leq \exp (\mathcal{A}(1))|B(0,1)| .
$$

Thus (53) holds.

To verify that $J(\cdot, f) \in L^{1}(B(0,1))$, we use the equation (58),

$$
\int_{G} J(x, f) d x \leq \sum_{i=1}^{\infty}\left|B_{i}\right|<\infty
$$

and the trivial estimate

$$
\int_{B(0,1) \backslash G} J(x, f)=|B(0,1) \backslash G| .
$$

Let us now explain how to choose the points $x_{i}, y_{i}$. Set $x_{i}=a_{i}$. If $y_{i} \in B_{i}$, then

$$
\begin{aligned}
\left|f\left(x_{i}\right)-f\left(y_{i}\right)\right| & =r_{i} \rho_{r_{i}}\left(\left|x_{i}-y_{i}\right|\right) \\
& =r_{i} \exp \left(-\int_{\left|x_{i}-y_{i}\right|}^{r_{i}} \frac{d s}{s \mathcal{A}^{-1}\left(\frac{n}{1+\epsilon_{i}} \log \frac{1}{s}\right)}\right) .
\end{aligned}
$$

Using the changes of variable $\mathcal{A}(t)=\frac{n}{1+k \epsilon_{i}} \log \frac{1}{s}, k=1,2$, we see that

$$
r_{i} \exp \left(-\int_{\left|x_{i}-y_{i}\right|}^{r_{i}} \frac{d s}{s \mathcal{A}^{-1}\left(\frac{n}{1+\epsilon_{i}} \log \frac{1}{s}\right)}\right) \geq i \exp \left(-\int_{\left|x_{i}-y_{i}\right|}^{1 / 2} \frac{d s}{s \mathcal{A}^{-1}\left(\frac{n}{1+2 \epsilon_{i}} \log \frac{1}{s}\right)}\right)
$$

when $y_{i}$ is chosen to be sufficiently close to $x_{i}$. The claim follows by noting that

$$
\mathcal{A}^{-1}\left(\frac{n}{1+\epsilon} \log \frac{1}{s}\right) \leq \mathcal{A}^{-1}\left(\frac{n}{1+2 \epsilon_{i}} \log \frac{1}{s}\right)
$$

when $2 \epsilon_{i} \leq \epsilon$.

\section{REFERENCES}

[1] David, G. (1988). Solutions de l'équation de Beltrami avec $\|\mu\|_{\infty}=1$. (French) [Solutions of the Beltrami equation with $\|\mu\|_{\infty}=1$ ] Ann. Acad. Sci. Fenn. Ser. A I Math. 13, no. 1, 25-70. MR 90d:30058

[2] Giannetti, F., Iwaniec, T., Onninen, J., and Verde, A. (2002). $L^{1}$-Estimates of Jacobians by subdeterminants. J. Geom. Anal. 12, no. 2, 223-254.

[3] Greco, L. (1998). Sharp integrability of nonnegative Jacobians. Rend. Mat. Appl. (7) 18, no. 3, 585-600. MR 2000g:42024

[4] Greco, L., Iwaniec, T. and Moscariello, G. (1995). Limits of the improved integrability of the volume forms. Indiana Univ. Math. J. 44, no. 2, 305-339. MR 96k:46051

[5] Hencl, S. and Malý, J., Mappings of finite distortion: Hausdorff measure of zero sets. Preprint. Charles University, 2001.

[6] Iwaniec, T., Koskela, P., Martin, G. J. and Sbordone, C., Mappings of finite distortion: $L^{n} \log ^{\alpha} L$-integrability. Preprint 246, Department of Mathematics and Statistics, University of Jyväskylä, 2001

[7] Iwaniec, T., Koskela, P. and Onninen, J. (2001). Mappings of finite distortion: Monotonicity and continuity. Inventiones Mathematicae 144, no. 3, 507-531. MR 2002c:30029 
[8] Iwaniec, T., Koskela, P. and Onninen, J. (2002). Mappings of finite distortion: Compactness. Ann. Acad. Sci. Fenn. Math. 27, no. 2, 391-417.

[9] Iwaniec, T. and Martin, G. J., The Beltrami equation, Mem. Amer. Math. Soc., to appear.

[10] Iwaniec, T. and Martin, G. J. (2001). Geometric function theory and non-linear analysis. Oxford Mathematical Monographs.

[11] Iwaniec, T. and Sbordone, C. (1992). On the integrability of the Jacobian under minimal hypotheses. Arch. Rational Mech. Anal. 119, no. 2, 129-143. MR 93i:49057

[12] Kauhanen, J., Koskela, P. and Malý, J. (2001). Mappings of finite distortion: Discreteness and openness. Arch. Rational Mech. Anal. 160, 135-151.

[13] Kauhanen, J., Koskela, P. and Malý, J. (2001). Mappings of finite distortion: Condition N. Michigan Math. J. 49, no. 1, 169-181. MR 2002d:30027

[14] Kauhanen, J., Koskela, P., Malý, J., Onninen, J. and Zhong, X., Mappings of finite distortion: Sharp Orlicz-conditions. Rev. Mat. Iberoamericana, to appear.

[15] Koskela, P. and Malý, J., Mappings of finite distortion: The zero set of the Jacobian. Preprint 241, Department of Mathematics and Statistics, University of Jyväskylä, 2001.

[16] Morrey, C. B. (1938). On the solutions of quasilinear elliptic partial differential equations, Trans. Amer. Math. Soc., 43, 126-166.

[17] Onninen, J., A note on the isoperimetric inequality. Proc. Amer. Math. Soc., to appear.

[18] Reshetnyak, Yu. G. (1967). Spatial mappings with bounded distortion. Sibirsk. Mat. Zh., 8, 629-659; English transl. in Siberian Math. J. 8 (1967). MR 35:6825

University of Jyväskylä, Department of Mathematics and Statistics, P.O. Box 35, Fin-40351 Jyvëskylä, Finland

E-mail address: pkoskela@maths.jyu.fi

University of Jyväskylä, Department of Mathematics and Statistics, P.O. Box 35, Fin-40351 JyvÄskylä, Finland

E-mail address: jaonnine@maths.jyu.fi 